$0,55 \%$ im Mittel beträgt, ist die Differenz bei dem in der Müble vermablenen Roggen bezw. dem zugehörigen Mehl und der zugehörigen Kleie viel größer, 2,8\% in Mittel. Daß diesen Ergebnissen nicht etwa ein Fehler der Berechnungsweise zugrunde liegt, geht schon ohne weiteres daraus hervor, dab das selbstermahlene Mehl einen kaum geringeren Stärkegehalt hat, wie das in der Mühle ermahlene, dabei aber die selbstermahlene Kleie infolge der Unvollkommenheit der Mahlvorrichtung die 2-3-fache Menge Stärke enthält wie die Kleje aus der Mühle.

Dieser Befund spricht dafür, daß 3 beim Vermahlen des Roggens in der Mühle ein picht unwesentlicher Teil der Stärke verschwunden, d. h. in den „Schwund" eingegangen ist, der in der Müllerei eine große Rolle spielt und bei den vorliegenden Vermahlungen in der Mühle bei Mehl I zu $4,9 \%$, bei II zu $2,5 \%$ und bei III $\mathrm{zu} 2,9 \%$ angegeben wurde.

Ein „Schwund" kann normalerweise nur durch Eintrocknen und Verstauben hervorgerufen werden. Der Einfluß des Austrocknens ist durch die Berechnung auf Trockensubstanz nusgeschaltet; es kann somit der gröBere Stärkeverlust in der Mühle nur darauf zurückgeführt werden, daß die Stärke in den „Staub“ gegangen ist.

\title{
Moste des Jahres 1921 aus den Weinbaugebieten der Nahe, des Glans, des Rheintals unterhalb des Rheingaues, des Rhein- gaues, der Lahn, des Rheins und Mains.
}

\author{
Von \\ Dr. J. Stern.
}

Mitteilung aus dem Nabrangsmitteluntersuchungsamt Kreuzach.

[Eingegangen am 25. April 1922.]

Es wurden 327 Moste untersucht, und zwar aus dem Bezirk des Amtes 205 Moste und aus dem Regierungsbezirk Wiesbaden 122 Moste. Hierunter waren 322 Weißund 5 Rotmoste.

Die Ergebnisse der Untersuchungen sind in den nachfolgenden Tabellen zusammengestellt.

I. Mostgewichte (Grade Oechsle).

1. Bezirk des Amtes.

\begin{tabular}{|c|c|c|c|c|c|c|c|c|c|c|c|c|c|c|c|}
\hline Weil & $\begin{array}{l}0 \\
+ \\
0 \\
1 \\
0 \\
0 \\
8 \\
0\end{array}$ & $\begin{array}{l}0 \\
8 \\
0 \\
1 \\
0 \\
0 \\
0\end{array}$ & $\left|\begin{array}{c}0 \\
+1 \\
1 \\
0 \\
0 \\
0\end{array}\right|$ & $\left|\begin{array}{c}0 \\
0 \\
0 \\
1 \\
0 \\
20 \\
2\end{array}\right|$ & $\begin{array}{l}0 \\
\dot{\alpha} \\
1 \\
1 \\
0 \\
0\end{array}$ & $\begin{array}{c}0 \\
8 \\
\infty \\
1 \\
0 \\
10 \\
\infty\end{array}$ & $\begin{array}{l}\sigma \\
\dot{8} \\
\dot{1} \\
\dot{8} \\
8\end{array}$ & $\begin{array}{l}0 \\
g \\
g \\
0 \\
0 \\
0 \\
0\end{array}$ & $\begin{array}{l}0 \\
\dot{0} \\
0 \\
1 \\
0 \\
8 \\
0\end{array}$ & $\begin{array}{l}0 \\
8 \\
0 \\
1 \\
0 \\
8 \\
0\end{array}$ & 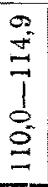 & $\mid \begin{array}{l}0 \\
\sigma \\
\exists \\
0 \\
0 \\
2 \\
=\end{array}$ & 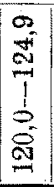 & $\begin{array}{l}0 \\
g \\
0 \\
0 \\
0 \\
0 \\
0\end{array}$ & 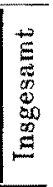 \\
\hline Nahe (Kreis Krenznach). . & - & - & 1 & 4 & 17 & 18 & 20 & 25 & 25 & 8 & 5 & 2 & 3 & 1 & 129 \\
\hline Glans und Nahe (Kreis Meisenheim) & 1 & 2 & - & 5 & 4 & 2 & 5 & 3 & - & - & - & - & - & - & 22 \\
\hline $\begin{array}{l}\text { Wheintal, linksrheinisch, unterhalb des } \\
\text { Rheivgaues (Kreis St, Goar) }\end{array}$ & - & 1 & 11 & 10 & 8 & 11 & 8 & 5 & - & - & - & - & -1 & - & 54 \\
\hline nsgesam & 1 & 3 & 12 & 19 & 29 & $\mid 31$ & 33 & 33 & 25 & 8 & 5 & 2 & 3 & 1 & 05 \\
\hline
\end{tabular}


2. Regierungsbezirk Wiesbaden.

\begin{tabular}{|c|c|c|c|c|c|c|c|c|c|c|c|c|c|c|c|}
\hline Weinbaugebiet & $\mid \begin{array}{c}0 \\
0 \\
1 \\
0 \\
0 \\
0\end{array}$ & $\begin{array}{l}9 \\
8 \\
1 \\
0 \\
0 \\
8\end{array}$ & $\begin{array}{c}0 \\
0 \\
1 \\
0 \\
0 \\
8\end{array}$ & 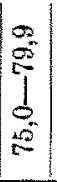 & $\begin{array}{l}0 \\
0 \\
1 \\
1 \\
0 \\
8\end{array}$ & $\begin{array}{c}9 \\
8 \\
0 \\
1 \\
0 \\
20 \\
20\end{array}$ & $\mid \begin{array}{c}0 \\
0 \\
1 \\
8 \\
8\end{array}$ & $\begin{array}{c}g \\
g \\
1 \\
0 \\
g \\
g\end{array}$ & $\left|\begin{array}{l}0 \\
0 \\
0 \\
0 \\
8 \\
0\end{array}\right|$ & $\left|\begin{array}{c}0 \\
g \\
0 \\
1 \\
0 \\
20 \\
9\end{array}\right|$ & $\mid \begin{array}{l}0 \\
\vdots \\
\vdots \\
0 \\
0 \\
\Xi\end{array}$ & $\begin{array}{l}0 \\
0 \\
0 \\
0 \\
=\end{array}$ & $\mid \begin{array}{l}0 \\
0 \\
0 \\
0 \\
0 \\
0 \\
0\end{array}$ & $\left|\begin{array}{c}0 \\
0 \\
2 \\
1 \\
1 \\
0 \\
2 \\
心\end{array}\right|$ & 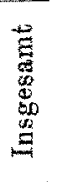 \\
\hline $\begin{array}{l}\text { heintal, rechtsreinisch, unter } \\
\text { Rheingaues (Kreis St, Goars }\end{array}$ & 1 & 1 & 1 & 2 & 8 & 5 & 7 & - & -1 & -1 & - & $1-$ & - & -1 & 25 \\
\hline$. \cdot . \cdot \cdot$. & - & - & - & 1 & 1 & 3 & 5 & 8 & 26 & 16 & 14 & 1 & - & 1 & 76 \\
\hline Oberlahnkreis $\cdot{ }^{*} \cdot \cdot \cdot \cdot \cdot \cdot \cdot$ & 1 & - & - & - & 2 & 1 & - & - & - & - & - & - & - & - & 4 \\
\hline $\begin{array}{l}\text { Weinlaaugebiet des Rheins und Mains } \\
\text { (Kreis Wiesbaden, Stadt und Land) }\end{array}$ & & -1 & - & - & - & - & 2 & 2 & 9 & 2 & 2 & - & - & -1 & 17 \\
\hline Ins: & 2 & $1)$ & 1 & 31 & 11 & 9 & 14 & 10 & 35 & 18 & 16 & 1 & -1 & 1 & \\
\hline
\end{tabular}

II. Freie Säure (g in $100 \mathrm{ccm}$ ).

1. Bezirk des Amtes.

\begin{tabular}{|c|c|c|c|c|c|c|c|c|c|c|c|}
\hline Weinbaugebiet & $\begin{array}{c}0 \\
0 \\
0 \\
0 \\
0 \\
0 \\
0\end{array}$ & $\begin{array}{c}9 \\
\mathbb{1} \\
0 \\
1 \\
0 \\
0 \\
0\end{array}$ & $\frac{8}{0}$ & $\begin{array}{l}8 \\
0 \\
0 \\
0 \\
0\end{array}$ & $\mid \begin{array}{c}R \\
0 \\
1 \\
2 \\
0 \\
0\end{array}$ & $\mid \begin{array}{c}8 \\
0 \\
0 \\
0 \\
0 \\
0\end{array}$ & $\left|\begin{array}{c}\mathscr{g} \\
0 \\
1 \\
8 \\
8 \\
0\end{array}\right|$ & $\begin{array}{l}8 \\
8 \\
1 \\
8 \\
8\end{array}$ & $\begin{array}{l}0 \\
-1 \\
1 \\
0 \\
0 \\
-2\end{array}$ & $\begin{array}{c}2 \\
-1 \\
1 \\
2 \\
- \\
-1\end{array}$ & 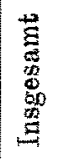 \\
\hline Nahe (Kreis Krenznach) . . . . . . . . . & 3 & 23 & 53 & 29 & 14 & 6 & 1 & - & - & -1 & 129 \\
\hline Glans und Nahe (Krois Meisenheim). . & - & 2 & 4 & 5 & 5 & 2 & 3 & - & - & 1 & 22 \\
\hline $\begin{array}{l}\text { Rheintal, linksrheinisch, unterhalb des Rheingaues } \\
\text { (Kreis St. Goar) }\end{array}$ & - & 3 & 9 & 9 & 20 & 9 & 4 & - & - & - & 54 \\
\hline lnsgesamt & 31 & 28 & 66 & 43 & 39 & 17 & 8 & $1-$ & - & $1 \mid$ & 205 \\
\hline
\end{tabular}

2. Regierangsbezirk Wiesbaden.

\begin{tabular}{|c|c|c|c|c|c|c|c|c|}
\hline Weinbaugebiet & 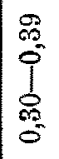 & 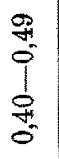 & $\begin{array}{l}0 \\
0 \\
0 \\
0 \\
0 \\
0 \\
0 \\
0\end{array}$ & $\begin{array}{l}0 \\
0 \\
0 \\
0 \\
0 \\
8 \\
8 \\
0\end{array}$ & $\sum_{0}^{\infty}$ & $\begin{array}{l}0 \\
\alpha \\
0 \\
0 \\
0 \\
0 \\
0 \\
0\end{array}$ & $\begin{array}{l}8 \\
\stackrel{8}{0} \\
\stackrel{8}{8} \\
8\end{array}$ & 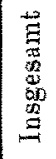 \\
\hline $\begin{array}{l}\text { Rheintal, rechtsrheinisch, unterhalb des Rheinganes } \\
\text { (Kreis St. Goarshausen) }\end{array}$ & 1 & 3 & 6 & 6 & 5 & 3 & 1 & 25 \\
\hline Rheingau . . . . . . . . . . . . . . & 10 & 28 & 24 & 13 & 1 & - & - & 76 \\
\hline Oberlahnkreis . . . . . . . . . . . . . . & - & - & 1 & 2 & - & 1 & - & 4 \\
\hline 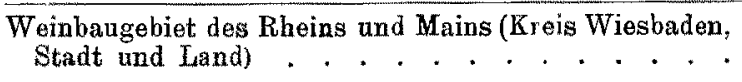 & - & 7 & 3 & 4 & 3 & - & - & 17 \\
\hline Insgesamt & 11 & 38 & 34 & 25 & 9 & 4 & 1 & 122 \\
\hline
\end{tabular}

Die Gesamtergebnisse werden in der amtlichen Weinstatistik $1921 / 22$ veröffentlicht werden. 
Es seien unter anderem folgende Befunde mitgeteilt:

\begin{tabular}{|c|c|c|c|c|c|}
\hline \multicolumn{2}{|c|}{ Rotmoste } & \multicolumn{3}{|c|}{ Bezirk des Amtes } & Reg.-Bez. Wiesbaden \\
\hline & höchstes & $88,4^{0}$ & $\begin{array}{l}\text { Oechsle bei } 4,9 \% / 00 \\
\text { (Portugieser) }\end{array}$ & Säure & $\begin{array}{c}118^{\circ} \text { bei } 4,4 \% \text { Säure } \\
\text { (Spätburgunder) }\end{array}$ \\
\hline Mostgewicht & niedrigstes & & (a) & & $\begin{array}{c}101^{\circ} \text { bei } 6,3^{\circ} / 00 \text { Säure } \\
\text { (Klebrot) }\end{array}$ \\
\hline
\end{tabular}

Weismoste

\begin{tabular}{|c|c|c|c|}
\hline Mo & $\left\{\begin{array}{l}\text { höchstes } \\
\text { niedrigstes }\end{array}\right.$ & $\begin{array}{r}\left.127,2^{0} \text { bei } 6,1^{0} \% 0 \text { Säure }{ }^{1}\right) \\
\left.64,6^{\circ} \text { bei } 12,4^{0} \text { oo Säure }{ }^{1}\right)\end{array}$ & $\begin{array}{r}128,2^{0} \text { bei } 5,8 \% \text { Säure } \\
\left.61,5^{0} \text { bei } 5,6^{\circ} \% \text { Säure } 1\right)\end{array}$ \\
\hline ت & $\left\{\begin{array}{l}\text { höchster } \\
\text { niedrigster }\end{array}\right.$ & $\begin{array}{r}\left.12,4^{\circ} \% \text { bei } 64,6^{01}\right) \\
4,0^{0} \% 0 \text { bei } 102,0^{01}\end{array}$ & $\begin{array}{l}\left.9,4^{0} / 00 \text { bei } 79,3^{02}\right) \\
\left.3,6^{0} \% \text { bei } 111,0^{03}\right)\end{array}$ \\
\hline
\end{tabular}

AuBerdem aus dem Bezirk des Amtes 13 Auslesemoste.

\begin{tabular}{|c|c|c|}
\hline & $\left\{\begin{array}{l}\text { höchstes } \\
\text { niedrigstes }\end{array}\right.$ & $\begin{array}{c}\left.294,7^{0} \text { bei } 14,9^{\circ} \% \text { Säure }{ }^{1}\right) \\
\left.189,2^{0} \text { bei } 10,6^{\circ} \% 0 \text { Säure }{ }^{4}\right)\end{array}$ \\
\hline & $\left\{\begin{array}{l}\text { höchster } \\
\text { niedrigster }\end{array}\right.$ & $\begin{array}{c}\left.16,0^{\circ} / 00 \text { bei } 247,4^{01}\right) \\
8,7^{\circ} \% \text { bei } 236,7^{0}\end{array}$ \\
\hline
\end{tabular}

Im Bezirk des Amtes ist die Weinernte 1921 an Menge sebr verschieden, an Güte durchweg ausgezeichnet ausgefallen. An der unteren Nahe und am Mittelrhein hatten viele Weinbergslagen sehr stark unter den Spätfrösten im Monat Mai zu leiden. Das Ernteergebnis war daher in diesen Lagen weit geringer als im Vorjahre. Durch das anhaltende heiße und trockne Wetter waren Krankheiten und Schädlinge des Weinstockes nur in bescheidenem Umfange zu beobachten; besonders der Heu- und Sauerwurm, welcher als Heuwurm im Monat Juni in vielen Lagen sehr stark aufgetreten war, wirkte als Sauerwurm in den Monaten August und September weit weniger schädigend. Ende September konnten die Trauben durchweg als vollreif betrachtet werden und so begannen auch viele Gemeinden schon in den ersten Tagen des Oktober mit der Weinlese. Die Mostgewichte spielten anfangs zwischen 90 und $100^{\circ}$ Oechsle, solche unter $90^{\circ}$ kamen weniger, vereinzelt allerdings auch solche unter $80^{\circ}$ vor. In erstklassigen Lagen waren Mostgewichte von $120-130^{\circ}$ keine Seltenheit. Durch Trockenbeer-Auslese erzielte das Weingut Graf von Plettenberg

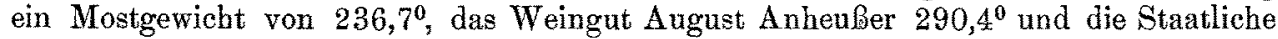
Weinbaudomäne Niederhausen-Schloßböckelbeim 294,70 Oechsle. Solche hohen Mostgewichte sind an der Nahe bisher nicht annähernd erzielt worden. Die freie Säure des Jahrganges 1921 war durchweg gering; doch dürfte der hohe Alkoholgehalt die Haltbarkeit der Weine auf der Flasehe garantieren. Mit ganz vereinzelter Ausnahme ist der Jahrgang 1921 natur gelegt worden und probieren sich die junge Weine als reife, vollmundige, zum Teil auch süPe Qualitätsweine. Hier und da findet man auch Jungweine, bei welchen der bohe Alkoholgehalt etwas einseitig hervorschmeckt. Die Mostpreise betrugen 13000-15000 M. je Stück. Zur Zeit (Ende April) werden die geringsten 1921-er Naturweine mit 40000-50000 M., mittlere Weine mit 60000 bis $70000 \mathrm{M}$ je Stück bezahlt, für Qualitätsweine werden Phantasiepreise angelegt.

Im Regierungsbezirk Wiesbaden zeitigte das Jahr 1921 eine Weinerte von ganz hervorragender Güte, einen Qualitätswein ersten Ranges. Nachdem der Austrieb der Reben schon frühzeitig erfolgt war, ging unter Wirkung der fast ohne Unterbrechung herrschenden trocknen, warmen Witterung auch die weitere Entwickelung der Weinstöcke mit ihrem zwar nicht großen, aber überall vorhandenen Traubenhang sehr schnell vor sich. Die günstige und schnelle Entwickelung ließen Krankheiten und Schädlinge nicht aufkommen. Durch das sonnige Wetter im Oktober wurde die

1) Riesling. 2) Gemischte Trauben. 3) Österreicher. ") Sylvaner. 
Qualität noch besonders gesteigert. Schon Anfang Oktober war eine Vollreife der Trauben erreicht. Hervorragende Produkte wurden in den Lagen mit tiefgründigen feuchten Böden erzielt, während die Berglagen infolge der Trockenheit weniger günstig abgeschnitten haben. Das Mengenergebnis ist im Durchschnitt etwas unter $1 / 2$ Ernte geblieben. Die Mostgewichte, beginnend mit den geringsten Lagen, überragen die in den guten Jahrgängen 1920, 1917, 1915, 1911 erzielten Mostgewichte aus den gleichen Lagen zum Teil ganz beträchtlich. Im Rheingau sind bei den Mosten, die aus Traubenverkäufen selbst von kleinen Winzern erzielt wurden, $100^{\circ}$ Oechsle oder nur wenige Grad darunter festgestellt worden, was seit Menschengedenken nicht beobachtet worden ist. Ein Unterschied gegenüber früheren guten Jahrgänge besteht insofern, als infolge des Mangels an Feuchtigkeit keine Edelfäule der Beeren eintrat und nicht die berühmten Spätlesen erzielt werden konnten. Die sogenannten Spitzen traten daher beim 1921-er weniger zahlreich und auffällig hervor. Der Säuregehalt ist sehr niedrig. Er bewegt sich im allgemeinen zwischen $5-6 \%$, geht in zahlreichen Fällen auf $4 \%$ und sogar bis 3,6\% berunter. Wäbrend die Preise im Herbst 25000-34000 M. für ein Stück Most betrugen, wurden anfangs Januar die 1921-er Weine mit 50000-70000 M., zur Zeit (Ende April) mit 15000G bis 200000 M. je Stück bezahlt; für die Spitzen werden ebenfalls Phantasiepreise angelegt.

\section{Feferate.}

\section{Allgemeine Bestandteile der Nahrungs- und Genubmittel.}

I. Arisz: Sol- und Gelzustand von Gelatinelösungen. (Kolloidchem. Beihefte 1915, 7, 1-90.) - Der erste Teil der Abhandlung befabu sich mit der Gelatini erung und bringt im ersten Kapitel Untersuchungen über die. Viskosität von Gelatinelösung in Glycerin unter dem Einfluß von Temperatur, Vorgeschichte, Ruhe und Bewegung der Lösungen; im zweiten Kapitel Untersuchungen über die Abbängigkei der Intensität des Ty ndall-Phänomens von der Temperatur; das dritte Kapitel enthält eine theoretische Betrachtung. Die Ergebnisse sind, wie folgt, zusammengefaßt: 1. Für die Viskositätsänderungen unterhalb $65^{\circ}$ unter konstanten äußeren Verhältuissen sind zwei Ursachen vorhanden. Die eine, ausschließlich mit der thermischen Vorgeschichte zusammenhängende, beruht auf der Tatsache, daß sich bei jeder Temperatur eir anderer Gleichgewichtszustand in der inneren Zusammensetzung einstellt, die andere ausschließlich davon, ob die Lösung in Ruhe gelassen oder bewegt war. Das Gleichgewicht in der inneren Zusammensetzung stellt sich bei hoher Temperatur rasch ein und bei niedriger viel langsamer. Die Änderungen, die im Gelzustand eintreten, schließen sich denen des Solzustandes an. Die Änderungen, die unabhängig von der thermischen Vorgeschichte in einem nicht bewegten Sole auftreten, sind bei niedriger Temperatur größer. Aus dem Verlauf der Viskositätsänderungen bei einer Temperatur, wobei schließlich Gelatinierung auftritt, läßt sich folgern, daß beim Übergang in den Gelzustand zwei Ursachen zusammenwirken. - 2. Die Intensität des Tyndall-Phänomens in einer Gelatinelösung hängt von der Temperatur, bei der sie untersucht wird, und von der thermischen Vorgeschichte ab. Bei Wärme ist sie klein, bei Kälte groß. Bei konstanter Temperatur tritt je nach der Vorgeschichte eine $\mathrm{Zu}$ nahme oder eine Abnahme der Intensität auf. Nur bei hoher Temperatur wird der Endzustand bald erreicht. Die Änderungen der Lichtintensität kommen in gleicher Weise zustande in Lösungen, die wohl und in solchen, die nicht gelatiniert sind. Bei Gelatinierung tritt nicht eine besonders starke Intensitätsänderung auf. In konzentrierten Lösungen erreichen die Intensitätsänderungen einen größeren Umfang als in 